\title{
Acute rheumatic fever: Findings of a hospital-based study and an overview of reported outbreaks
}

UPTON D ALLEN, MB,BS, FAAP, FRCPC, MICHAEL BRAUDO, MBCHB, FRCP(EDIN), STANLEY E READ, MD, PHD, FAAP, FRCPC

\begin{abstract}
To review the characteristics of reported outbreaks of acute rheumatic fever in the United States, and to determine if there is an increase in the incidence of acute rheumatic fever in the population served by the Hospital for Sick Children. Toronto, Ontario, the authors conducted a literature search and a retrospective review of inpatients and outpatients, satisfying the revised Jones criteria for the diagnosis of acute rheumatic fever, from 1972 to 1988 . Patients satisfying the revised Jones criteria for the time period 1972-88 were included in the study. There have been eight articles reporting an increase in acute rheumatic fever in the United States. In three, the majority of children were white and from middle class suburban/rural communities in different geographic locations. Mucoid strains of group A streptococci were implicated but not confirmed as being associated with the outbreaks in three. The results of the chart review at the Hospital for Sick Children revealed that 83 cases satisfied the revised Jones criteria. The number of cases per 100,000
\end{abstract}

children (aged 18 years or less) per year, decreased progressively over the study period. Polyarthritis was the most frequently seen major criterion occurring in $73 \%$ of patients (61 of 83). The most frequently affected ethnic groups were Italians 23\%, Afro-Canadians 19\% and Orientals $8 \%$. The reported outbreaks in the United States are multifocal and predominantly confined to white middle class children residing in suburban/rural communities. There was no evidence of an increase in the number of cases of acute rheumatic fever seen in the population served by the Hospital for Sick Children; there was a progressive decline in number of cases over the study period. The results facilitate the characterization of acute rheumatic fever within North America into three different patterns of occurrence. Can J Infect Nis 1990; 1(3):77-81

Key Words: Acute rheumatic fever, Jones criteria, Outbreaks. Pharyngitis, Streptococcal infection
Divisions of General Pediatrics and Infectious Diseases. The Hospital for Sick Children. Toronto. Ontario

Correspondence and reprints: Dr Stanley Read. Division of Infectious Diseases. The Hospital for Sick Children. 555

University Avenue. Toronto. Ontario. M5G 1X8. Telephone (416) 598-6268

This paper was presented in part at The Canadian Pediatric Society Conference. Halifax. Nova Scotia. June 1989 1990
VER THE PAST 25 YEARS THERE HAS BEEN A progressive decline in the incidence of acute rheumatic fever in North America and other developed countries (1-6). In the United States, this decline has been associated with a marked reduction in morbidity and mortality attributable to acute rheumatic fever (7). Recent reports have described outbreaks of acute rheumatic fever in several areas in the United States (8-15) and Europe (16). While the disease remains of major concern in the developing countries, as well as in certain low income communities within North America $(2,17,18)$, many of these recent reports did not originate from areas of poverty or areas lacking access to medical care (8-11). In addition, it is of concern that several laboratories in the United States have been reporting increasing isolates of mucoid strains of group A streptococci $(19,20)$. While several factors contribute to the 
virulence of these organisms in man (21), such strains have been associated with increased virulence (19). Any increase in the incidence of acute rheumatic fever would be of great importance to all cosmopolitan North American cities, including Toronto.

The objective of this study was to review the experience in Toronto in the context of the North American experience in general. To facilitate this, the evidence for an increase in the incidence of acute rheumatic fever in North America was first examined. In addition, in the absence of any report of a resurgence of acute rheumatic fever in Toronto, all cases of acute rheumatic fever from 1972 to 1988 inclusive at the Hospital for Sick Children in Toronto were reviewed to determine if there was any evidence for an increase in incidence in the population served by the hospital.

\section{METHODS}

Literature review: MEDLINE searches were conducted for the period 1985-89, in order to obtain all reports of outbreaks in the continental United States. In addition, an extensive manual search of the bibliographies of primary reports, review articles and editorials was conducted. Any report of an increase of acute rheumatic fever in North America since 1985 was considered for inclusion; unpublished reports were not considered.

Data from relevant studies were synthesized and examined in detail with respect to methodological, clinical, epidemiological, social and demographic characteristics.

Review at the Hospital for Sick Children: The Hospital for Sick Children is a 587 bed hospital and Canada's largest pediatric institution. In 1972 there were 166,407 outpatient visits, while in 1988 there were 267,932 such visits (22). The hos ital is situated in metropolitan Toronto and is the only tertiary pediatric hospital in Toronto and adjoining suburban areas, serving a local population of 415,000 children aged 18 years or less living in the borough of East York and the cities of Etobicoke, North York, Scarborough, Toronto and York (23). (These five cities and one borough are collectively referred to as 'metropolitan Toronto.') It also provides secondary and primary care for a significant proportion of the pediatric population of metropolitan Toronto.

The medical records of both inpatients and outpatients diagnosed as having acute rheumatic fever during 1972-88 inclusive were reviewed. The list of patients was generated from the medical records department, and to ensure completeness, the authors screened the charts of all patients seen during the study period with a diagnosis of acute rheumatic fever, rheumatic heart disease, rheumatic carditis or Sydenham's chorea. Patients were considered to have acute rheumatic fever if they met the revised Jones criteria (24). This requires that the patient manifest two major criteria - carditis, polyarthritis, chorea, erythema marginatum or subcutaneous nodules - or one major and two minor criteria, with evidence of antecedent streptococcal infection. The diagnosis is also suggested by the presence of chorea. In addition to clinical and laboratory data, each record was reviewed for social and demographic data.

All patients with acute rheumatic fever had been seen by a cardiology consultant (including $\mathrm{MB})$, who confirmed the presence of the various criteria. The interaction between cardiologists, general pediatricians and rheumatologists was such that questionable cases were seen by all three services and a consensus reached regarding the diagnosis of acute rheumatic fever. Patients were followed in a special acute rheumatic fever clinic by MB. Clinic data were obtained from the Cardiology Data Centre and reviewed, and clinic personnel consulted to ensure that no recent cases of acute rheumatic fever were missed. This also enabled verification of the information obtained from the medical records department.

Patients were excluded if acute rheumatic fever was diagnosed outside of the study period; if they presented during the study period with old rheumatic heart disease; or if they were diagnosed outside of Ontario.

Incidence rates were calculated using census data for metropolitan Toronto obtained from the metropolitan planning department. The number of children younger than 18 years of age as well as the population percentages attributable to the respective ethnic groups were obtained. (The 1981 census was used for the ethnic percentages, because this information was not available for the other census years.)

\section{RESULTS}

Literature review: There were eight articles reporting outbreaks of acute rheumatic fever in the continental United States (8-15). Five were from tertiary pediatric institutions (8-12), two from military bases $(13,14)$ and one from a general hospital centre (15). The reports were from centres in different geographic locations. The number of cases reported and the corresponding baselines (where available) are shown in Table 1. This shows a difference between study and control periods for those reports that documented baseline values. Though the study by Veasy et al (10) did not provide baseline data for Salt Lake City, the rate of 18.1 per 100,000 reported was noted to be about 


\section{TABLE 1}

Reported outbreaks of acute rheumatic fever in the United States: Summary of study characteristics and results

\begin{tabular}{|c|c|c|c|c|c|c|c|c|}
\hline Reference & 8 & 9 & 10 & 11 & 12 & 13 & 14 & 15 \\
\hline Design & $P / R$ & $R$ & $R$ & $R$ & $R$ & $R$ & $R$ & $R C$ \\
\hline Medical setting & Ped & Ped & Ped & Ped & Ped & Army & Navy & Gen \\
\hline Location & Akr & Pitt & SLC & Colum & Nash & FLW & SDN & NYC \\
\hline Control period & $1976-85$ & $1965-84$ & $1975-85$ & $1974-83$ & $1985-86$ & - & $1982-86$ & $1975-85$ \\
\hline Number of cases & 23 & 17 & $74^{\dagger}$ & 40 & 26 & 14 & 10 & 6 \\
\hline Cases/100,000 (control) & - & - & $\ldots$ & - & - & - & 0.75 & - \\
\hline
\end{tabular}

PProspective: RRetrospective review: RC Retrospective case reports: Ped Tertiary pediatric centre: Gen General hospital: Akr Akron, Ohio: Pitt Pittsburgh. Pennsylvania: SLC Salt Lake City, Utah: Colum Columbus, Ohio: Nash Nashville, Tennessee: FLW Fort Leonard Wood, Missouri: SDN San Diego Navy. California; NYC New York City. New York. "Study period was from January to June 1985. 67 resided in Utah, three in ldaho. two in Nevada and two in Wyoming. ${ }^{\dagger}$ Incidence reported for 26 children from Salt Lake City seen in $1985 .{ }^{\S}$ Incidence for control period not available. Incidence reported was about 10 times greater than previous highest rate for comparable United States locations (10)

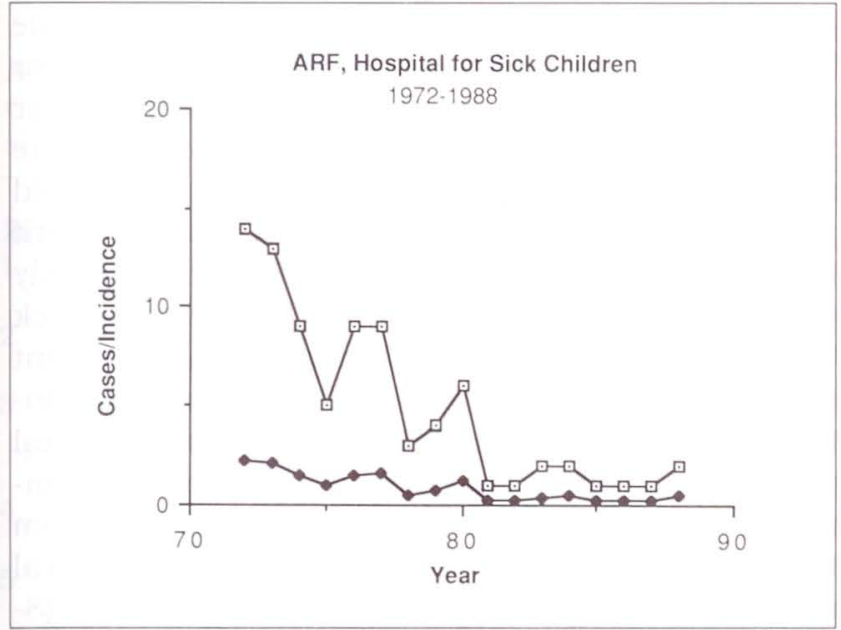

Figure 1) Declining incidence of acute rheumatic fever at the Hospital for Sick Children in Toronto. Ontario. 1972 88. Open squares Cases: Blackened squares Incidence

10 times greater than the previous highest reported United States rate and was comparable to rates of 30 years before.

Social and demographic data revealed that for the studies with complete data, the cases were predominantly among whites. In three reports (810) there was clear documentation that the majority resided in suburban or rural areas. The results of four complete reports showed that the majority of cases were from homes with above average family income and/or with adequate health insurance coverage (8-11).

In one study, mucoid $\mathrm{M}$ type 18 and $\mathrm{M}$ type 3 group A streptococci were isolated from some siblings and from a sample of school children (10). In another, $74 \%$ of group A streptococcal isolates obtained from a survey were M type 18 and 20\% M type 3 (14), while in Columbus, Ohio increasing numbers of M type 18 were observed (11).
Chart review: During the period 1972-88 inclusive, 83 cases satisfied the revised Jones criteria for the diagnosis of acute rheumatic fever. Sixty-two cases were seen during the first seven years of the study period 1972-78 inclusive, while 21 were seen during the 10 year period 1979-88 inclusive. There were 49 males and 34 females (male/female ratio 1.4/1). Ages ranged from three to 18 years, with a mean age of 11 years. During the first half of the study period, the ratio of children aged 10 years or less to those greater than 10 years old was $1 / 1.2$. The same ratio for the second half of the study period was $1 / 2$, thus indicating proportionately more older children presenting with acute rheumatic fever during the 1980s.

Figure 1 shows a progressive downward trend in the number of cases seen each successive year. The corresponding rates per 100,000 children aged 18 years or less showed a progressive decline over the study period. In addition, the results also indicated a decrease in the incidence of acute rheumatic fever within any age subgroup (three to seven, eight to 13 and 14 to 18 years, respectively). The highest recorded incidence was 2.2 cases per 100,000 children aged 18 years or less in 1972, while the incidence in 1988 was 0.50 per 100,000 .

The most frequent major criterion manifested was polyarthritis in $73 \%$ of patients (61 of 83) (Table 2). Carditis occurred in 52\% (43 of 83), chorea in $7 \%$ (six of 83), subcutaneous nodules in $6 \%$ (five of 83 ), and erythema marginatum in $4 \%$ (three of 83). Chorea was the sole major criterion in five of six patients with chorea, while one patient had all five major criteria including chorea.

Minor criteria manifested are shown in Table 2. Fever and arthralgia were documented in 94\% (78 
of 83 ) and $66 \%$ (55 of 83) of cases, respectively. An elevated erythrocyte sedimentation rate (greater than $20 \mathrm{~mm} / \mathrm{h}$ ) was documented in $88 \%$ (73 of 83), while a prolonged PR interval (greater than 18 ms) occurred in $31 \%$ (26 of 83 ).

Group A streptococci were isolated from the pharynx of $22 \%$ of children (18 of 83), while $54 \%$ (45 of 83) had a history of antecedent pharyngitis. One child had an antecedent history of scarlet fever. Results of streptococcal serotyping were not available, as this was not done on any isolate.

There were no deaths directly related to complications of acute rheumatic fever during the acute period. Complete follow-up records were available for 70 children. These revealed that $43 \%$ (30 of 70) had documented residual valvular dysfunction of varying degrees. The most common valvular lesion was mitral valve incompetence.

Social and demographic data relating to the 83 cases showed the following percentages for the most frequently affected ethnic groups: Italians $23 \%$, Afro-Canadians 19\%, Orientals 8\% and native Canadians $2 \%$. Census data (1981) revealed that the population percentages attributable to these groups were $11,0.7,3.9$ and $0.5 \%$, respectively (23). All but five children were resident in Canada for more than one year prior to diagnosis. A positive family history was documented in five cases. Family size was documented in 72 cases. The average family size for these cases was 5.2, compared with an average family size of 3.4 for metropolitan Toronto (based on 1981 statistics) (23).

\section{DISCUSSION}

The reports of recent outbreaks of acute rheumatic fever from the United States show a multifocal pattern, with white middle income families predominantly affected. Several reports have limitations with respect to the provision of baseline incidences and population trends. Nevertheless, it is clear that acute rheumatic fever remains a major concern to public health authorities in the United States. The extent to which the United States trend applies to Canada is not well documented.

In this regard, a review of cases of acute rheumatic fever seen at the Hospital for Sick Children in Toronto showed no evidence of an increase in the number of cases in the population served by the hospital. In fact, there has been a progressive decline in the number of cases seen over the study period. This occurred against a background of increasing population (23). In interpreting the results, one should bear in mind that the study did not include patients seen at smaller suburban hospitals or those seen by
TABLE 2

Clinical presentation of acute rheumatic fever at the Hospital for Sick Children, 1972-88

\begin{tabular}{lc}
\hline Criteria & Cases $(\%)$ \\
\hline Major criteria & \\
Polyarthritis & 73 \\
Carditis & 52 \\
Chorea & 7 \\
Subcutaneous nodules & 6 \\
Erythema marginatum & 4 \\
Minor criteria & \\
Fever & 94 \\
Arthralgia & 66 \\
ESR $>20 \mathrm{~mm} / \mathrm{h}$ & 88 \\
PR>0.18 ms & 31 \\
Previous ARF/RHD & 8 \\
\hline ESR Eryth
\end{tabular}

ESR Erythrocyte sedimentation rate: ARF Acute rheumatic fever: RHD Rheumatic heart disease

general pediatricians and not referred to the Hospital for Sick Children. The trend has been for such patients to be referred to the Hospital for Sick Children. An increase in incidence of acute rheumatic fever in metropolitan Toronto would probably have been associated with a concomitant increase in the number of cases seen at the only pediatric hospital in Toronto, the Hospital for Sick Children. The overall results concur with a recent study using data generated from the Ontario Ministry of Health computer database on hospital separations (25). The situation in other communities in Canara may be quite different from that in Toronto, as evidenced by the multifocal nature of the outbreaks in the United States (815). However, a survey of Canadian cardiologists did not suggest a resurgence of acute rheumatic fever in Canada (26).

The important morbidity caused by acute rheumatic fever is borne out by the fact that at least 36\% (30 of 83) of children in the present study population had varying degrees of detectable residual valvular damage. The relative order of occurrence of polyarthritis and carditis, the two most frequent major criteria, was similar to other series $(1,11,18)$. In one outbreak, carditis occurred in $91 \%$ of cases (10). This relatively high frequency of carditis is in part related to the use of echocardiography to diagnose carditis. Indeed, in the above study, the frequency of carditis was found to be $72 \%$ when diagnosed using auscultation (10).

Physicians should be aware of the importance of accurate diagnosis and treatment of group A streptococcal pharyngitis. In the Akron, Ohio outbreak, 30\% of patients received inadequate therapy for group A streptococcal pharyngitis (8). The present results show that $54 \%$ of children had a history of antecedent pharyngitis, while group A 
streptococcus was isolated from the pharynx of $22 \%$. The presence of subclinical group A streptococcal infection is of concern, as in the reported outbreaks only 21 to $55 \%$ of cases had documented pharyngitis (9-11).

The results showed a disproportionately high number of cases in children of Italian background, Afro-Canadians, Orientals and native Canadians. The reasons for this observation have not been established in this study. While the data available were not sufficient to conclude that overcrowding or any other specific factor was responsible, it should be noted that the average family size of 5.2 is 1.5 times greater than the average family size for metropolitan Toronto. It should also be noted that in the 1980s, acute rheumatic fever is still quite prevalent within some groups in North America, particularly those with lower socio-

ACKNOWLEDGEMENTS: Dr Allen is supported by a Canadian Infectious Diseases Society/Eli Lilly Fellowship.

\section{REFERENCES}

1. Disciascio G. Taranta A. Rheumatic fever in children. Am Heart J 1980;99:635-58.

2. Markowitz M. The decline of rheumatic fever: Role of medical intervention. J Pediatr 1985; 106:525-50.

3. Schollin J, Wesstrom G. Acute rheumatic fever in Swedish children. Acta Paediatr Scand 1985; 74:749-54.

4. Sievers J, Hall P. Incidence of acute rheumatic fever. Br Heart J 1971:33:833-6.

5. Bisno AL. Acute rheumatic fever: Forgotten but not gone. N Engl J Med 1987;316:476-8. (Edit)

6. Ferrieri P. Acute rheumatic fever: The come-back of a disappearing disease. Am J Dis Child 1987; 141:725-7.

7. Massell BF, Chute CG, Walker AM, et al. Penicillin and the marked decrease in morbidity and mortality from rheumatic fever in the United States. N Engl J Med 1988;318:280-6.

8. Congeni B, Rizzo C, Congeni J, et al. Outbreak of acute rheumatic fever in northeast Ohio. J Pediatr 1987;111:176-9.

9. Wald ER, Dashefsky B, Feidt C, et al. Acute rheumatic fever in western Pennsylvania and the Tristate area. Pediatrics 1987:80:371-4.

10. Veasy GL, Wiedmeier SE, Orsmond GS, et al. Resurgence of acute rheumatic fever in the intermountain area of the United States. N Engl J Med 1987:316:421-7.

11. Hosier DM, Craenen JM, Teske DW, et al. Resurgence of acute rheumatic fever. Am J Dis Child 1987:141:730-3.

12. Westlake RM, Graham TP. Edwards KM. An outbreak of acute rheumatic fever in Tennessee. Pediatr Infect Dis J 1990:9:97-100.

13. Centers for Disease Control. Acute rheumatic fever at a Navy Training Center-San Diego, California. economic status $(2,17,18)$. It is important to appreciate, however, that the recent outbreaks in the United States were not confined to the ethnic groups that have been traditionally affected.

In summary, the distribution of acute rheumatic fever within North America may be considered as existing in three patterns. One pattern describes disease occurrence in lower socioeconomic groups in inner cities and native communities. Another refers to epidemic occurrence of the disease in middle class children residing in suburban/urban communities. The third pattern refers to the sporadic occurrence of cases in low prevalence areas such as Toronto. Further studies are warranted to better characterize these patterns of occurrence, particularly with respect to the significance of mucoid streptococci, in order to facilitate preventive strategies.

MMWR 1988;37:101-4.

14. Centers for Disease Control. Acute rheumatic fever among trainees - Fort Leonard Wood, Missouri, 1987-1988. MMWR 1988:37:519-22.

15. Giardina A-CV, Heaton S. Acute rheumatic fever in New York City. N Y State J Med 1988;88:385-6.

16. Bonora G, Rogari P, Acerbi I, et al. Outbreak of acute rheumatic fever in northern Italy. J Pediatr 1989; 1 14:334. (Lett)

17. Longstaffe S. Postl B. Kao H, et al. Rheumatic fever in native children in Manitoba. Can Med Assoc J 1982;127:497-8.

18. Brownell KD. Bailen-Rose F. Acute rheumatic fever in children: Incidence in a borough of New York City. JAMA 1973:224:1593-7.

19. Kaplan EL. Return of rheumatic fever: Consequences, implications and needs. J Pediatr 1987:1 11:244-6. (Edit)

20. Kaplan EL, Johnson DR, Cleary PP. Group A streptococcal serotypes isolated from patients and sibling contacts during the resurgence of rheumatic fever in the United States in the mid-1980s. J Infect Dis 1989;159:101-3.

21. Wannamaker LW. Virulence factors in streptococci. Scand J Infect Dis 1982;31 (Suppl):22-7.

22. The Hospital for Sick Children. Annual reports, Hospital for Sick Children. Toronto. Canada. 1972- 1989.

23. Metropolitan Planning Department. Census and Demographic Data, Statistics Canada, 1971-1988.

24. Ad Hoc Committee to Revise the Jones Criteria (Modified), Council on Rheumatic Fever and Congenital Heart Disease of the American Heart Association. Jones criteria (revised) for guidance in the diagnosis of rheumatic fever. Circulation 1984;69:204A-8A.

25. Hutten-Czapski P. Acute rheumatic fever - No epidemic in Ontario. Can J Public Health 1989:80:7 1-2.

26. Hutten-Czapski P. Bass MJ. Is there a resurgence of acute rheumatic fever in Canada? Can Fam Phys 1989:35:1761-4. 


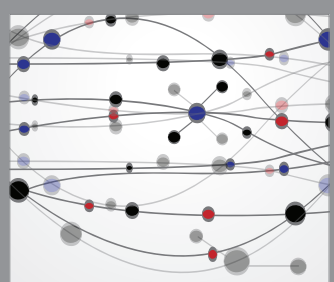

The Scientific World Journal
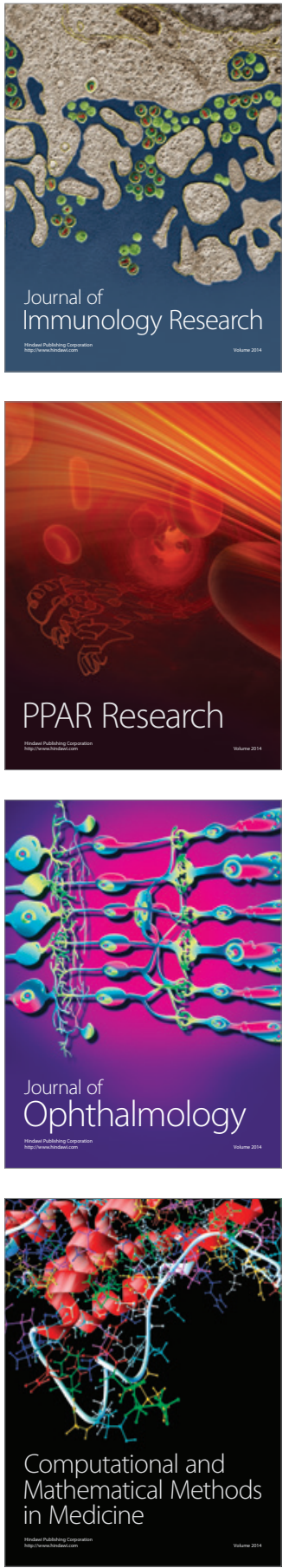

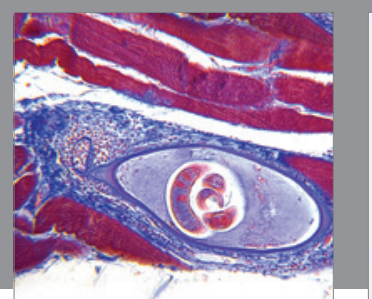

Gastroenterology Research and Practice

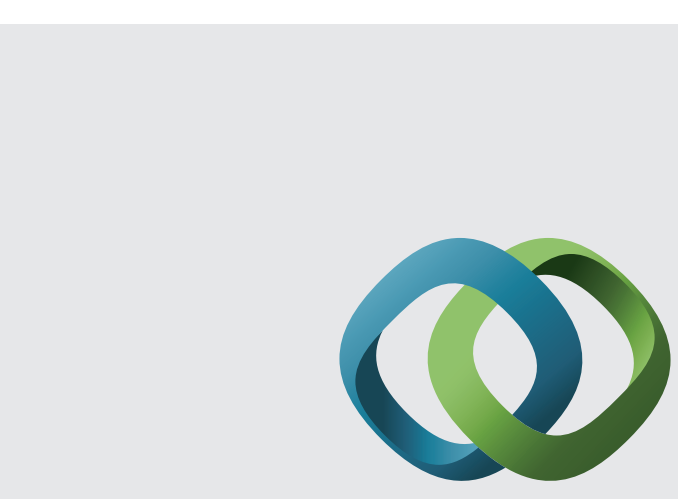

\section{Hindawi}

Submit your manuscripts at

http://www.hindawi.com
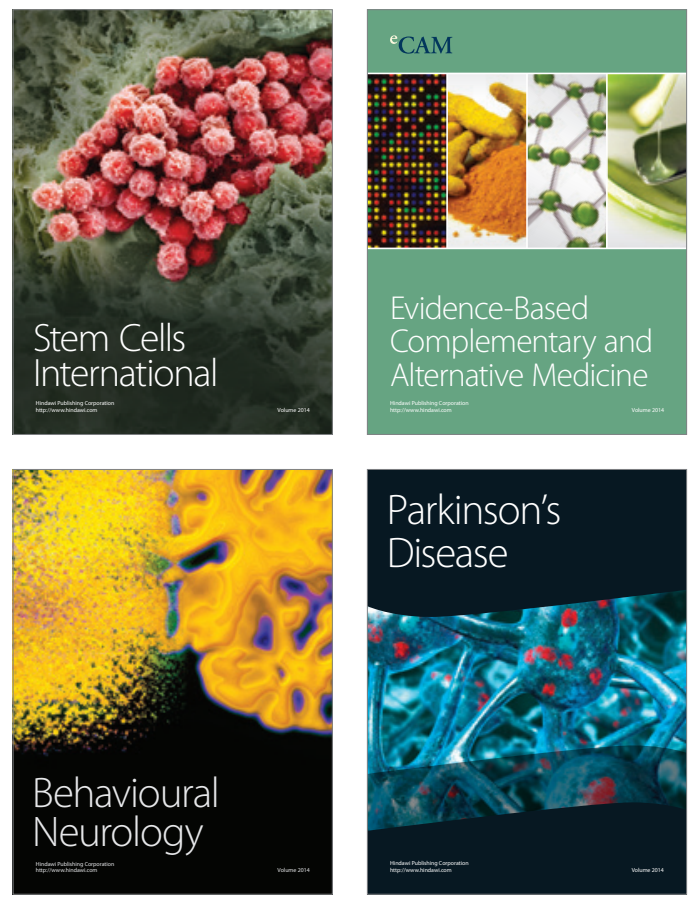
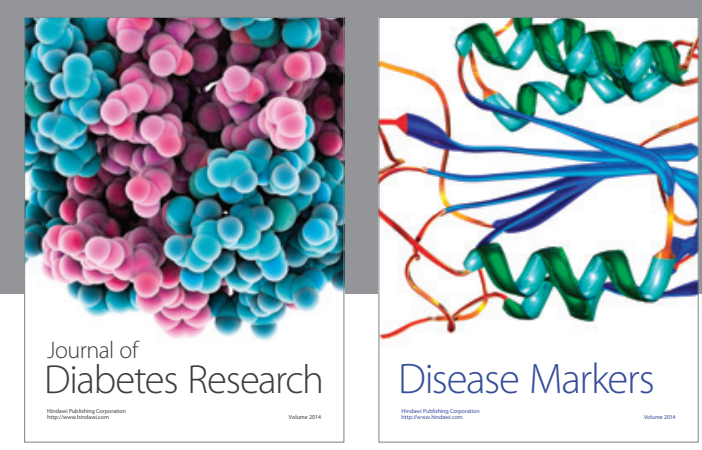

Disease Markers
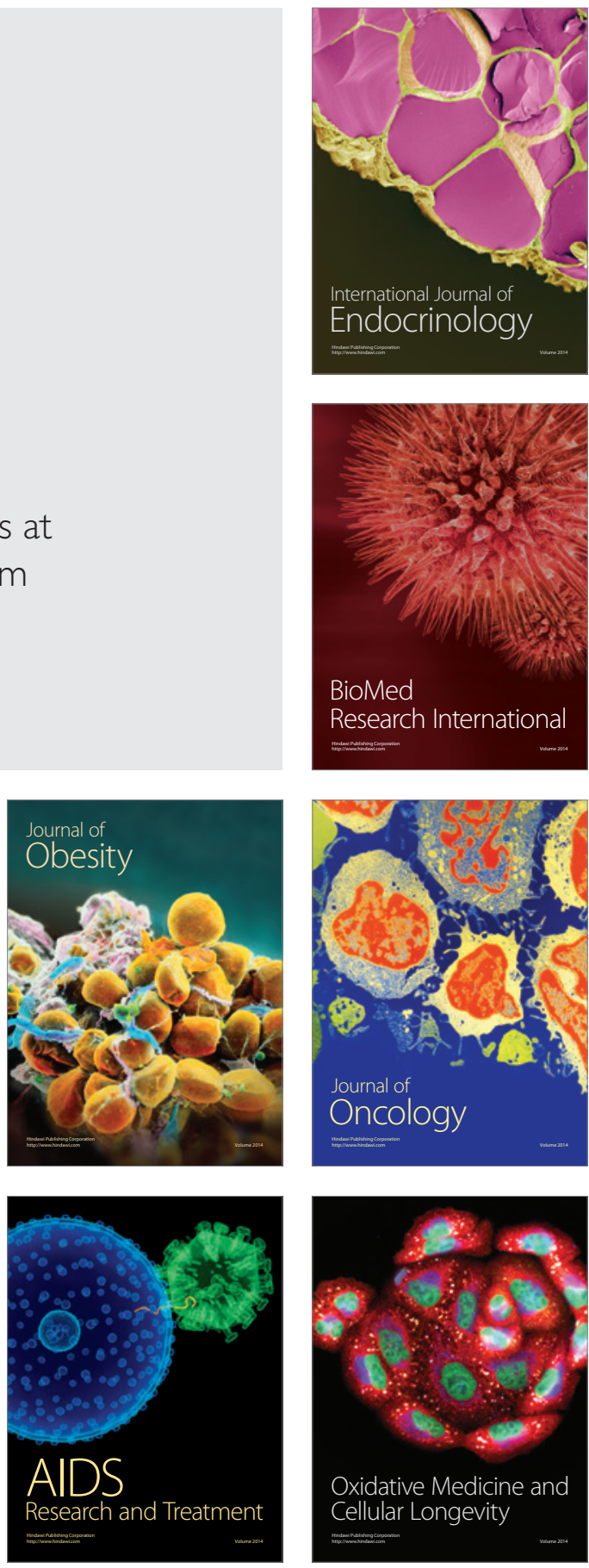